\title{
The Potential Impact of Morality on Medical Student Global Health Participation
}

\author{
Joel Rowe ${ }^{1} \&$ Stephen G. Post ${ }^{2}$ \\ ${ }^{1}$ Renaissance School of Medicine at Stony Brook University, NY 11794, USA \\ ${ }^{2}$ Center for Medical Humanities, Compassionate Care \& Bioethics, Renaissance School of Medicine at Stony \\ Brook University, NY 11794, USA \\ Correspondence: Joel Rowe, MD, Renaissance School of Medicine at Stony Brook University, NY 11794, USA. \\ E-mail: joelrsrowe@gmail.com
}

Received: August 1, 2019 Accepted: September 30, 2019 Online Published: November 3, 2019

doi:10.5539/gjhs.v11n13p1 URL: https://doi.org/10.5539/gjhs.v11n13p1

\begin{abstract}
Interest in global health experiences (GHEs) has surged in the last decade throughout undergraduate medical education. Positive clinical and cultural learning impacts are well described; however, the moral and motivational typology of the globally minded medical student are yet to be elucidated. We surveyed 85 US medical students, 41 who participated in a GHE during medical school and 44 who did not, to examine their sense of moral association with local community, Americans, and all of humanity. Measures of empathy and spirituality were also administered, as well as a qualitative prompt to elicit reasons for participating, or not, in a GHE. The results of logic regression analysis suggest that the strongest predictors of GHE participation are strong, geographically non-specific identification with 'all humanity' [OR=1.31, $\mathrm{P}<0.01,95 \% \mathrm{CI}, 1.07-1.59$ ], as well as participation in an abroad experience prior to medical school [OR 141, $\mathrm{P}<0.01,95 \% \mathrm{CI}, 10.1-1960$ ]. While respondent groups did not differ significantly in their association with local community, incremental increase in identification with 'Americans' decreased likelihood of IME participation by $20 \%$ [P=0.02, 95\% CI, 0.67-0.96]. No significant effect was found between participant groups in response to empathy or spirituality scales. This pilot study demonstrates that a global regard for 'all humanity' may motivate GHE participation while a strong national association diminishes its likelihood.
\end{abstract}

Keywords: attitudes/values, burnout, global health, medical education, medical humanities

\section{Introduction}

Interest in global health experiences (GHEs) among medical trainees and educators in the US continues to rise. At the undergraduate level, one-fourth of medical students participate in a GHE and more than two-thirds of allopathic medical schools support a global health student interest group (Khan et al., 2013; Walker, Campbell, \& Egede, 2015). Myriad positive outcomes for GHE learners have been identified, including improved confidence with the history and physical exam, heightened cross-cultural awareness, greater appreciation for preventive services, and an increased disposition to care for the medically underserved (Bruno, Imperato, \& Szarek, 2014; Jeffrey, Dumont, Kim, \& Kuo, 2011; Thompson, Huntington, Hunt, Pinsky, \& Brodie, 2003). Accordingly, students pursue GHEs for educationally practical reasons: to learn to care for patients in low resource environments, build collaborative relationships with international organizations, and integrate global health into their future careers (Leow et al., 2012; Moren et al., 2015).

Yet to date, little attention has been paid to the moral motivations of the globally minded medical student. In an environment of physician shortage in parts of the US and in typically referent nations of the term 'global' health, the question of which populations a doctor in training directs his or her moral compass becomes immediate. Certainly, no hierarchy of 'good' is appropriate based on where a volunteer gives of their time. Yet, among a student population presumably selected for their altruistic tendencies, why some direct their sentiment globally and others more locally sheds light on how individuals in a generation of physicians might operationalize concepts like globalization and the inevitability of a shrinking global village.

We examined whether a geographically agnostic sense of global consciousness itself motivates medical students to participate in a GHE. To this end, we investigated a medical student's self-reported moral commitment to the three 
domains of 'local community,' 'all Americans,' and/or 'all humanity' and how this association might inform abroad participation. Additional plausible driving forces, including spirituality and empathy, were also investigated. Many definitions of religion and spirituality include notions of giving and universal kindness and both such organizations are well represented in the global health arena. Whether GHE participation represents a heightened expression of trainee empathy was also questioned.

\section{Method}

\subsection{Participant Characteristics}

Medical students (MD and DO) enrolled in a United States institution with written competency in English were eligible to take part in the study. Participants from the Stony Brook University School of Medicine $(n=75,88.2 \%)$, as well as clinical student volunteers (US MD students from multiple institutions) at the non-profit medical mission Floating Doctors in Bocas del Toro, Panama $(n=10,11.8 \%)$ participated in the study.

\subsection{Sampling Procedures}

Stony Brook medical students (Classes of 2019, 2018, and 2017; 390 students; response rate 19.2\%) received recruitment emails containing a confidential Qualtrics survey link. Floating Doctors clinical volunteers were approached in person and offered the opportunity to complete either an online or paper survey. Responses to paper surveys were digitized into the Qualtrics survey by manual entry.

\subsubsection{Sample Size}

A total of 85 US MD students completed the survey.

\subsubsection{Measures}

The survey contained questions from three well established and verified scales: (a) the Identification With All Humanity Scale (IWAH) (McFarland, Webb, \& Brown, 2012), (b) the Jefferson Scale for Physician Empathy (JSPE) (Fields et al., 2011), and (c) the Daily Spiritual Experience Scale (DSES) (Underwood, 2011). Demographic information, past international humanitarian experience (prior to medical school), estimated education debt, and desired medical specialty were also collected. A brief qualitative prompt (response limited to two sentences) to elicit reasons for participation in an GHE, or not, was simultaneously administered.

\subsubsection{Research Design}

Participants (Table 1) were categorized as: students who participated in an GHE at any point during medical school (GHE, $n=41,48.2 \%$ ), and students who did not participate in an GHE during medical school (Non-GHE, $n=44$, 51.8\%). Approval for survey administration and data collection/analysis was granted by the Stony Brook University Institutional Review Board.

\section{Results}

\subsection{Recruitment}

Participants were recruited via email from the Stony Brook University School of Medicine ( $n=75,88.2 \%)$, as well as in person from clinical student volunteers (US MD students from multiple institutions) at the non-profit medical mission Floating Doctors in Bocas del Toro, Panama $(n=10,11.8 \%)$ in June-July 2016. Stony Brook medical students received a total of three recruitment emails containing a confidential Qualtrics survey link once every two weeks for six weeks.

\subsection{Statistics and Data Analysis}

Scale data was analyzed through bivariate t-testing and linear regression modeling to compare variables which might influence GHE participation. Data were analyzed using SPSS Version 25 for Windows (Armonk, NY). Results of bivariate t-testing revealed a statistically significant relationship $(\mathrm{P}<0.01)$ between GHE participation and high response to the 'all humanity' measure of the IWAH scale (Table 2). The use of binary logistic regression then allowed for comparison of the impact of multiple variables (scale scoring, age, gender, desired field, estimated education debt, and pre-med abroad participation) on whether students participated or not in a GHE during medical school (Table 3). This analysis suggests that incremental response increase on the 'all humanity' measure of the IWAH scale corresponds with increased likelihood of GHE participation by $31 \%$ [P $<0.01,95 \%$ CI, $1.07-1.59]$. This effect remained significant despite the remarkably strong impact $(\mathrm{OR}=141)$ of pre-med abroad participation on later GHE participation in medical school [P $<0.01,95 \%$ CI, 10.1-1960]. By contrast, incremental increase in participant response on the 'Americans' measure corresponded to a $20 \%$ decrease $[\mathrm{P}=0.02,95 \% \mathrm{CI}$, 0.67-0.96] in likelihood of GHE participation. Age, gender, and estimated education debt were not found to impact GHE participation. 
No significant effect was found between participant groups in response to JSPE or DSES scales; likewise, respondent groups did not vary significantly in their association with local community or with 'all Americans' in bivariate t-testing (Table 2).

Participant narratives were sorted for the presence of themes and their corresponding frequency. The most frequently noted barrier to GHE participation in narrative responses (Table 4$)$ was cost $(n=15,34.1 \%)$. The most frequently cited reasons for GHE participation were a desire for a break from school $(n=14,34.1 \%)$ and to learn about a new culture $(n=13,31.7 \%)$.

\section{Discussion}

\subsection{Predictors of Participation}

Our pilot survey reveals that a sense of globally shared or 'common' humanity beyond geographic proximity is statistically predictive of GHE participation, while stronger association with 'Americans' significantly decreases this likelihood (Rowe, Post, \& Messina, April 22, 2017). This suggests that medical students who participate in GHEs may be motivated by a moral concern for others that transcends in-group preference or immediate geographic location. Further, that a strong sense of association with 'Americans' predicted a decreased likelihood of GHE participation is noteworthy as the tension between globalism and nationalism continues to pervade contemporary political discourse.

We cannot anticipate if and how medical students will manifest their globalism in practice. But it is worth noting that physicians who participate in global health work at the attending level have been categorized into three main groups: the globalized doctor (most common), who works primarily in his or her home community, but who elects to regularly serve abroad; the humanitarian doctor primarily based in and practicing outside their home country; and the policy doctor who works on the issues effecting the health of patients around the world (Rowson et al., 2012). Global consciousness may manifest across a medical student's career in different ways depending on circumstances and timing, and as familial and other special relationships evolve through life and further training. But as a matter of intentionality and conscience, it is ethically important that some individuals lean outwards towards a non-specific all humanity, rather than directing focus towards their local community.

Whatever the precise manifestation, that previous volunteer participation strongly begets medical school GHE participation is unsurprising. A recent survey of attending physicians found that $60-85 \%$ of those who participated in international medical missions during residency training continued similar work once fully trained. By contrast, only $6-7 \%$ of doctors who did not work internationally during residency later volunteered abroad for the first time at the attending level (Tannan \& Gampper, 2015). An acculturation process, perhaps compounding with additional abroad experiences, may be at play. One area for further study would be a longitudinal investigation of IWAH responses, rather than a single snapshot in time, to ask whether a critical response threshold exists at which a respondent will transition to GHE participation. This could also be used to assess whether identification with 'all humanity' increases or decreases through the course of medical training and could capture more medical students who participate in a GHE for the first time in the senior portions of their undergraduate education.

The lack of statistically meaningful difference in spirituality and empathy responses suggests empathy can be equally expressed through relationships with in-groups, or through non-specific expression outward to rest of the globe. Globalists appear to be no more spiritual or 'holier than thou' in our small, self-reporting sample. Rather, to act in the global domain may be an expression of a universally shared human sentiment that some students choose to inculcate early into their professional identity formation, perhaps even prior to the start of their undergraduate medical education.

In favor of increasing IMEs, many have argued that the potential of meaningful partnerships to impact areas in which need far outstrips the supply of healthcare goods and services provides mutual benefit for the host community and its volunteers (Palazuelos \& Dhillon, 2016). Tenets of successful programs have been shown to include broad institutional support, strong mentorship, generation of standardized competencies, and adequate pre-departure training for students and providers to reduce potential harm and maximize benefit to host communities (Nelson, Saltzman, \& Lee, 2012; Scott, 2013; Smith \& Weaver, 2006). The barriers fully trained providers face to working internationally has only begun to appear in research literature, including identification of a 'Global Health Tax' on their goodwill (Palazuelos \& Dhillon, 2016). Rarely, if ever, is this type of work compensated monetarily in an equal way to work performed stateside.

Yet service abroad is one way some medical students may extend a geographically non-specific desire to care for others to its perceived farthest reaches and in so doing, reconnect with the altruistic sentiment that compelled them to reach for a stethoscope. Physicians, meanwhile, rightfully complain of spending more time working at 
computers than with patients, of being overworked, and understaffed. In pilot studies, doctors find lasting rejuvenation in IMEs where they can enjoy reconnecting with both patients and their own higher motivations of altruistic care (Iserson, 2018). Short-term global medical experiences are increasingly shown to improve a provider's mental health and reinvigorate an underlying passion to practice medicine. What's more, the effects appear sustained at over a year post-intervention (Campbell et al., 2009; Iserson, 2018). The plausibility of employing IMEs to ameliorate burnout and reengage physicians with their work and sense of mission domestically is a fascinating topic to build on this pilot study. Examining whether GHE participation and domestic volunteerism have a protective or ameliorative impact on the eventual burnout indices of pilot participants would be worthwhile.

\subsection{Study Limitations}

Limitations of this pilot study include a small sample size of 85 respondents and a low response rate of Stony Brook medical students (19.2\%). The latter may be attributable to email-based recruitment to this population, which comprised the majority of the study sample (88.2\%). Bias may also have been injected through the recruitment of a large number of GHE participants (24.4\%) from one clinical volunteer site (Floating Doctors). Our regression model may also not have captured all the potential influences on a medical student's decision to participate in an GHE. We limited our scope to the predictive value of scale responses intended to capture elements of the moral typology that may drive medical students to participate in GHEs. Factors other than independent variables of our analysis (age, estimated education debt, desired specialty field, and pre-med abroad participation) may impact a medical student's decision to participate in an GHE. Follow-up study could integrate the most oft-cited qualitative responses in a similar regression model to weigh their impact relative to the moral measures we examined.

Additionally, the significance of 'Other' desired specialty field is difficult to meaningfully interpret as it represents an amalgam of inexact (e.g. multiple fields, or 'primary care,' a field reachable through multiple medical residencies) and low-frequency responses. Free response was utilized for this survey question in order to capture the common uncertainty among MS2 and MS3 students with regard to specialty selection.

\section{Conclusions}

It is no secret in medicine that the emergence of new infections, increased international travel, and climate change all require providers to care for diverse patient populations regardless of the setting in which they train (Landrigan et al., 2011). Many, if not most major healthcare settings are irreversibly cosmopolitan and increasingly globally diverse. Much needs to be learned about GHE involvement with regard to motive and potential benefits to the medical students and physicians seeking sustained meaning and self-care, as well as to host populations. That students may harvest internal benefits in this performance of global altruism abroad is a plausible hypothesis to be further explored.

Medical students widely fear loss of empathy in a depersonalizing healthcare system (Eisenstein, 2018), and indeed this may be one reason why travel to distant sites to interact with patients in indigenous settings remains cathartically appealing. Perhaps GHEs can be offered to enhance resilience among medical trainees and clinicians who seek to regain meaning in their practice through empathic communication with patients as they express a common sense of shared humanity (Center et al., 2003; Gordon, Baranov, \& Fleisher, 2018; Kline, Harford, \& Culp, 2018).

\section{Acknowledgements}

Special thanks to Catherine R. Messina, $\mathrm{PhD}$, for her statistical expertise and contagious kindness. A warm thank you to the founder and CEO of Floating Doctors, Benjamin LaBrot, MD, for his project support and dedicated service to the indigenous populations of Panama.

\section{Competing Interests Statement}

The authors declare that there are no competing or potential conflicts of interest.

\section{References}

Bruno, D. M., Imperato, P. J., \& Szarek, M. (2014). The correlation between global health experiences in low-income countries on choice of primary care residencies for graduates of an urban US medical school. $J$ Urban Health, 91(2), 394-402. https://doi.org/10.1007/s11524-013-9829-4

Campbell, C., Campbell, D., Krier, D., Kuehlthau, R., Hilmes, T., \& Stromberger, M. (2009). Reduction in burnout may be a benefit for short-term medical mission volunteers. Mental Health, Religion \& Culture, 12(7), 627-637. https://doi.org/10.1080/13674670903124541 
Center, C., Davis, M., Detre, T., Ford, D. E., Hansbrough, W., Hendin, H., . . Silverman, M. M. (2003). Confronting depression and suicide in physicians: a consensus statement. Jama, 289(23), 3161-3166. https://doi.org/10.1001/jama.289.23.3161

Eisenstein, L. (2018). To Fight Burnout, Organize. New England Journal of Medicine, 379(6), 509-511. https://doi.org/10.1056/NEJMp1803771

Fields, S. K., Mahan, P., Tillman, P., Harris, J., Maxwell, K., \& Hojat, M. (2011). Measuring empathy in healthcare profession students using the Jefferson Scale of Physician Empathy: Health provider -- student version. Journal of Interprofessional Care, 25(4), 287-293 287p. https://doi.org/10.3109/13561820.2011.566648

Gordon, E. K., Baranov, D. Y., \& Fleisher, L. A. (2018). The role of feedback in ameliorating burnout. Curr Opin Anaesthesiol, 31(3), 361-365. https://doi.org/10.1097/aco.0000000000000583

Iserson, K. V. (2018). Burnout Syndrome: Global Medicine Volunteering as a Possible Treatment Strategy. $J$ Emerg Med, 54(4), 516-521. https://doi.org/10.1016/j.jemermed.2017.12.062

Jeffrey, J., Dumont, R. A., Kim, G. Y., \& Kuo, T. (2011). Effects of international health electives on medical student learning and career choice: results of a systematic literature review. Fam Med, 43(1), 21-28.

Khan, O. A., Guerrant, R., Sanders, J., Carpenter, C., Spottswood, M., Jones, D. S., . . Wright, S. (2013). Global health education in U.S. medical schools. BMC Med Educ, 13, 3. https://doi.org/10.1186/1472-6920-13-3

Kline, A. D., Harford, J. S., \& Culp, M. P. (2018). Does Involvement in Global Health Reduce Perceived Radiologist Burnout? J Am Coll Radiol, 15(8), 1196-1198. https://doi.org/10.1016/j.jacr.2017.10.031

Landrigan, P. J., Ripp, J., Murphy, R. J., Claudio, L., Jao, J., Hexom, B., . . Koplan, J. P. (2011). New academic partnerships in global health: innovations at Mount Sinai School of Medicine. Mt Sinai J Med, 78(3), 470-482 https://doi.org/10.1002/msj.20257

Leow, J. J., Groen, R. S., Kingham, T. P., Casey, K. M., Hardy, M. A., \& Kushner, A. L. (2012). A preparation guide for surgical resident and student rotations to underserved regions. Surgery, 151(6), 770-778. https://doi.org/10.1016/j.surg.2012.03.002

McFarland, S., Webb, M., \& Brown, D. (2012). All humanity is my ingroup: A measure and studies of identification with all humanity. Journal of Personality and Social Psychology, 103(5), 830-853. https://doi.org/10.1037/a0028724

Moren, A., Cook, M., McClain, M., Doberne, J., Kiraly, L., Perkins, R. S., \& Kwong, K. (2015). A pilot curriculum in international surgery for medical students. $J$ Surg Educ, 72(4), e9-e14. https://doi.org/10.1016/j.jsurg.2015.04.027

Nelson, B. D., Saltzman, A., \& Lee, P. T. (2012). Bridging the global health training gap: Design and evaluation of a new clinical global health course at Harvard Medical School. Med Teach, 34(1), 45-51. https://doi.org/10.3109/0142159x.2011.577122

Palazuelos, D., \& Dhillon, R. (2016). Addressing the "Global Health Tax" and "Wild Cards": Practical Challenges to Building Academic Careers in Global Health. Acad Med, 91(1), 30-35. https://doi.org/10.1097/acm.0000000000000845

Rowe, J., Post, S. G., \& Messina, C. (April 22, 2017). Examining Medical Student Motivations to Explore Experiences in Global Health. Paper presented at the Yale Global Health \& Innovation Conference, New Haven, CT.

Rowson, M., Smith, A., Hughes, R., Johnson, O., Maini, A., Martin, S., . . Yudkin, J. S. (2012). The evolution of global health teaching in undergraduate medical curricula. Global Health, 8, 35. https://doi.org/10.1186/1744-8603-8-35

Scott, J. (2013). Medical student in global health-just one part of a larger commitment. Acad Med, 88(11), 1596-1597. https://doi.org/10.1097/ACM.0b013e3182a705d1

Smith, J. K., \& Weaver, D. B. (2006). Capturing medical students' idealism. Ann Fam Med, 4 Suppl 1, S32-37; discussion S58-60. https://doi.org/10.1370/afm.543

Tannan, S. C., \& Gampper, T. J. (2015). Resident Participation in International Surgical Missions is Predictive of Future Volunteerism in Practice. Arch Plast Surg, 42(2), 159-163. https://doi.org/10.5999/aps.2015.42.2.159

Thompson, M. J., Huntington, M. K., Hunt, D. D., Pinsky, L. E., \& Brodie, J. J. (2003). Educational effects of international health electives on U.S. and Canadian medical students and residents: a literature review. Acad 
Med, 78(3), 342-347.

Underwood, L. G. (2011). The Daily Spiritual Experience Scale: Overview and Results. Religions, 2(1), 29.

Walker, R. J., Campbell, J. A., \& Egede, L. E. (2015). Effective strategies for global health research, training and clinical care: a narrative review. Glob J Health Sci, 7(2), 119-139. https://doi.org/10.5539/gjhs.v7n2p119

\section{Appendix}

Table 1. Demographics and Pre-Med Abroad Experience

\begin{tabular}{lccccc}
\hline Group & Freq & Male & Female & Mean Age (SD) & Pre-Med Abroad \\
\hline IME & 41 & $15(37 \%)$ & $26(63 \%)$ & $24.7(2.11)$ & $24(59 \%)$ \\
Non-IME & 44 & $20(45 \%)$ & $24(55 \%)$ & $25.3(2.47)$ & $4(9 \%)$ \\
\hline
\end{tabular}

Table 2. Identification with All Humanity (IWAH) scale, Jefferson Scale for Physician Empathy (JSPS), Daily Spirituality Experience Scale (DSES), results of bivariate t-testing.

\begin{tabular}{lllclc}
\hline Scale & IME $(\mathbf{n}=\mathbf{4 1})$ & SD & Non-IME $(\mathbf{n}=\mathbf{4 4})$ & SD & Significance \\
\hline IWAH & & & & & \\
Community & 37.4 & 7.34 & 37.1 & 7.18 & 0.87 \\
Americans & 34.1 & 7.21 & 35.1 & 5.75 & 0.46 \\
All Humanity & $\mathbf{3 6 . 8}$ & $\mathbf{5 . 0 5}$ & $\mathbf{3 3 . 6}$ & $\mathbf{5 . 5 1}$ & $<\mathbf{0 . 0 1 *}$ \\
JSPE & 24.0 & 2.67 & 24.0 & 2.77 & 0.87 \\
DSES & 32.4 & 7.52 & 29.8 & 7.36 & 0.11 \\
\hline
\end{tabular}

Table 3. Results of logistic regression analysis, including IWAH scale, age, gender, desired field, estimated education debt, and pre-med abroad experience, on IME participation

\begin{tabular}{llccc}
\hline Variable & Freq & Adj OR & $\mathbf{9 5 \%}$ CI & Significance \\
\hline Community Score & 85 & 0.99 & $0.87,1.13$ & 0.87 \\
Americans Score & 85 & $\mathbf{0 . 8 0}$ & $\mathbf{0 . 6 7 , 0 . 9 6}$ & $\mathbf{0 . 0 2 *}$ \\
All Humanity Score & 85 & $\mathbf{1 . 3 1}$ & $\mathbf{1 . 0 7 , 1 . 5 9}$ & $\mathbf{0 . 0 1}^{*}$ \\
\hline Age & 85 & 0.93 & $0.66,1.33$ & 0.70 \\
\hline Gender & & & & 0.43 \\
Male & 35 & 0.49 & $0.08,2.91$ & \\
Female (ref) & 50 & 1 & & 0.46 \\
\hline Desired Field & & & & 0.28 \\
Pediatrics & 14 & 2.17 & $0.28,16.8$ & 0.93 \\
Internal Medicine & 12 & 0.22 & $0.01,3.35$ & 0.07 \\
Emergency & 12 & 0.90 & $0.08,10.7$ & $\mathbf{0 . 0 2 *}$ \\
Surgery & 10 & 0.09 & $0.01,1.19$ & \\
Other & 14 & $\mathbf{0 . 0 4}$ & $\mathbf{0 . 0 0 , 0 . 6 0}$ & \\
Undecided (ref) & 15 & 1 & & 0.10 \\
\hline Estimated Debt & & & & 0.10 \\
\$200,000+ & 33 & 8.05 & $0.67,96.3$ & \\
\$1-199,000 & 37 & 7.24 & $0.71,74.4$ & \\
\$0 (ref) & 15 & 6 & & \\
\hline & & & & \\
& & 6 & & \\
\hline
\end{tabular}




\section{Pre-Med Abroad}

Yes

No (ref)
25

60
141

1
$10.1,1960$

$<0.01 *$

Table 4. Qualitative responses to, "In three sentences or less, please describe your motivation for pursuing or not pursuing international humanitarian work during your medical education.”

Top responses for participating in an GHE (frequency):

-want to travel/break from school (14)

-to learn about new cultures/gain perspective on belief systems (13)

-learn more about healthcare outside the US (9)

-serve underserved communities (8)

-learn a new language/increase competency in a foreign language (6)

-specific program opportunity/research (6)

Top responses for not participating* in an GHE (frequency):

-funding concerns/cost prohibitive/impact on debt (15)

-project/research at home institution (9)

-prefer to/committed to serving underserved populations in the US (7)

-relationship or family commitments/do not wish to be separate from partner (7)

*14 respondents noted interest in GHE participation (generally or in the future) but cited current obstacle(s).

\section{Copyrights}

Copyright for this article is retained by the author(s), with first publication rights granted to the journal.

This is an open-access article distributed under the terms and conditions of the Creative Commons Attribution license (http://creativecommons.org/licenses/by/4.0/). 